\title{
B-cell-depleting Therapy in Systemic Lupus Erythematosus
}

\author{
Manuel Ramos-Casals ${ }^{a}$, Iñaki Sanz ${ }^{b}$, Xavier Bosch $^{c}$, John H. Stone ${ }^{d}$, and Munther A. \\ Khamashta ${ }^{\mathrm{e}}$ \\ aLaboratory of Autoimmune Diseases Josep Font, IDIBAPS, Department of Autoimmune \\ Diseases, Hospital Clínic, Barcelona, Spain \\ bDivision of Allergy, Immunology and Rheumatology, Department of Medicine, University of \\ Rochester, School of Medicine and Dentistry, Rochester, NY \\ 'Department of Internal Medicine, ICMiD, Hospital Clínic, Barcelona, Spain \\ ${ }^{\mathrm{d} R h e u m a t o l o g y}$ Unit, Massachusetts General Hospital, Boston \\ 'Lupus Research Unit, The Rayne Institute, St. Thomas' Hospital, King's College, London, UK
}

\begin{abstract}
The emergence of a new class of agents (B-cell-depleting therapies) has opened a new era in the therapeutic approach to systemic lupus erythematosus, with belimumab being the first drug licensed for use in systemic lupus erythematosus in more than 50 years. Four agents deserve specific mention: rituximab, ocrelizumab, epratuzumab, and belimumab. Controlled trials have shown negative results for rituximab, promising results for epratuzumab, and positive results for belimumab. Despite these negative results, rituximab is the most-used agent in patients who do not respond or are intolerant to standard therapy and those with life-threatening presentations. B-celldepleting agents should not be used in patients with mild disease and should be tailored according to individual patient characteristics, including ethnicity, organ involvement, and the immunological profile. Forthcoming studies of B-cell-directed strategies, particularly data from investigations of off-label rituximab use and postmarketing studies of belimumab, will provide new insights into the utility of these treatments in the routine management of patients with systemic lupus erythematosus.
\end{abstract}

\section{Keywords}

Belimumab; Epratuzumab; Ocrelizumab; Rituximab; Systemic lupus erythematosus

\begin{abstract}
Systemic lupus erythematosus, a disease that predominantly affects young women and that may cause severe organ impairment and even death, is considered paradigmatic of systemic autoimmune diseases. ${ }^{1}$ The treatment of systemic lupus erythematosus remains a challenge because a balance must be sought between the demonstrated efficacy of immunosuppressive agents (mostly used off-label) and the adverse effects of immunosuppression. For the first
\end{abstract}

\footnotetext{
(C) 2012 Elsevier Inc. All rights reserved.
}

Requests for reprints should be addressed to Xavier Bosch, MD, PhD, Department of Internal Medicine, Hospital Clínic, Villarroel 170, Barcelona 08036, Spain., xavbosch@ clinic.ub.es.

Authorship: All authors had access to the data and played a role in writing this manuscript.

Conflict of Interest: JHS has received research funding from Genentech and Biogen-IDEC and has served as a consultant for the development of B-cell depletion strategies in the treatment of antineutrophil cytoplasmic autoantibody-associated vasculitis. IS has served as consultant for MedImmune, GSK, Pfizer, BMS, Takeda Pharmaceuticals, and Biogen. The remaining authors declare no conflict of interest. 
time in more than 50 years, the United States Food and Drug Administration and the European Medicines Agency have licensed a new drug for use in systemic lupus erythematosus: belimumab, a biological therapy targeting B lymphocytes. This suggests that a new era may be opening in our therapeutic approach to systemic lupus erythematosus, based on new drugs with more specific mechanisms of action. ${ }^{2}$ It seems an opportune moment for a practical update on this new class of drugs in systemic lupus erythematosus.

\section{B-CELL DEPLETION IN LUPUS TODAY}

\section{Current Agents}

B-cell therapies are designed to eliminate either the majority of B cells (general depletion) or only some B-cell populations (selective depletion) ${ }^{3}$ (Table 1 ). In both cases, depletion is achieved through 2 principal mechanisms:

1. Direct killing by monoclonal antibodies against B-cell surface molecules CD19, CD20 (rituximab, ocrelizumab), and CD22 (epratuzumab). The most widely tested category of anti-B-cell agents is anti-CD20 antibodies, which induce a broad and deep B-cell depletion.

2. Attrition due to inhibition of B-cell survival factors BLyS (belimumab) and APRIL (atacicept). Belimumab has a significantly more restricted and attenuated B-cell effect $^{4,5}$ by blocking the essential survival effect of BLyS. Atacicept induces the depletion of a significantly larger swathe of B cells and plasma cells, although this powerful effect also may increase the risk of severe infections.

\section{Results of Controlled Trials}

Rituximab-Two randomized controlled trials (RCTs) have evaluated the use of rituximab in patients with systemic lupus erythematosus (Table 2). The Study to Evaluate the Efficacy and Safety of Rituximab in Patients With Severe Systemic Lupus Erythematosus (EXPLORER) trial included 257 patients with moderate-severe nonrenal systemic lupus erythematosus. ${ }^{6}$ Patients were randomized to the addition of rituximab $(n=169)$ or placebo $(n=88)$ to the baseline immunosuppressive agents, together with a 10-week course of highdose glucocorticosteroids. The 2 arms of the trial showed no statistically significant reduction in clinical activity compared with baseline, and the hypothesized superiority of rituximab plus standard of care (SOC) over SOC alone was not demonstrated. The second RCT was the Study to Evaluate the Efficacy and Safety of Rituximab in Subjects With ISN/ RPS Class III or IV Lupus Nephritis (LUNAR) trial, a phase III trial that included 144 patients with proliferative lupus nephritis. The definitive results are not yet published, but preliminary results 7,8 show that the trial did not achieve its primary and secondary endpoints (Table 1). The failures of EXPLORER and LUNAR taught the community of lupus investigators valuable lessons about clinical trial design in this condition and influenced the development of subsequent lupus trials.

Ocrelizumab-The Study to Evaluate Ocrelizumab in Patients With Nephritis Due to Systemic Lupus Erythematosus (BELONG) trial tested ocrelizumab in patients with lupus nephritis with a design similar to that of the LUNAR trial. In March 2010, Roche (Basel, Switzerland) and Biogen (Cambridge, Mass) decided to suspend the ongoing trials of ocrelizumab in patients with rheumatoid arthritis and systemic lupus erythematosus following the recommendations of an independent monitoring board. The board had detected an infection-related safety signal (including severe and opportunistic infections), several of which were fatal, among the 2400 patients from more than 30 countries. The recently reported details of the BELONG trial ${ }^{9}$ showed a trend to a better response in the ocrelizumab $400 \mathrm{mg}(62 \%)$ and $1000 \mathrm{mg}(64 \%)$ arms in comparison with the placebo arm 
$(51 \%, P=.075)$. The percentage of patients experiencing serious infections was twice as high in patients who received concomitant mycophenolate (32\% vs $16 \%$ in the placebo arm). A specific geographical distribution of severe infections was detected in Asian patients. $^{9}$

Epratuzumab-The first trials of epratuzumab in systemic lupus erythematosus were terminated early due to difficulties in supplying the active agent. However, the results from 55 patients enrolled showed that epratuzumab-treated patients required smaller quantities of glucocorticosteroids when compared with placebo-treated patients over 24 weeks. ${ }^{10,11}$ Preliminary results of the 12-week Epidemiology of Burkitt Lymphoma in East Africa Children or Minors (EMBLEM) trial, a phase IIB RCT including 227 patients, have shown a clinical response of 38\% (epratuzumab $600 \mathrm{mg}$ weekly) and 35\% (epratuzumab $1200 \mathrm{mg}$ weekly) in comparison with the placebo arm $(22 \%){ }^{12}$

Belimumab—Clinical trials of belimumab in systemic lupus erythematosus began inauspiciously, with failure of a dose-ranging phase II trial of 449 patients to achieve its primary outcome. ${ }^{5}$ However, the trial included $30 \%$ of patients who had no antinuclear antibodies at baseline, raising questions about the validity of their systemic lupus erythematosus diagnoses. A subsequent analysis of a continuation trial in 296 of these 449 patients found that immunologically positive patients treated with belimumab showed sustained improvement in disease activity and a decrease in flares over 6 years of follow-up, accompanied by a reduction in glucocorticosteroid use. ${ }^{13}$

The recently published results of the Study of Belimumab in Subjects With Systemic Lupus Erythematosus (BLISS-52) trial marked the first positive RCT of a biologic agent in systemic lupus erythematosus (Table 2). This trial included 865 patients with positive immunological markers and moderate-severe disease. ${ }^{14} \mathrm{~A}$ clinical response at 52 weeks was achieved by $44 \%$ of placebo-treated patients compared with $51 \%$ of those receiving belimumab $1 \mathrm{mg} / \mathrm{kg}$ and $58 \%$ of those treated with belimumab $10 \mathrm{mg} / \mathrm{kg}(P=.013$ and . 0006, respectively), with modest but consistent improvements across a range of clinical outcome measures. A second trial (BLISS-76) included 819 patients with a similar design, although patients and investigators remained blinded for an additional 24 weeks (Table 2). The advance results at 52 weeks showed that the percentage of patients achieving a clinical response was $34 \%$ with placebo, $41 \%$ with $1 \mathrm{mg} / \mathrm{kg}$, and $43 \%$ with $10 \mathrm{mg} / \mathrm{kg}(P=.10$ and $P$ $=.021$, respectively) ${ }^{15}$ Analysis of the combined 1864 patients in both BLISS trials at 52 weeks shows reductions in disease activity and prevention of worsening in internal organ involvement. ${ }^{16}$ Superiority in the BLISS trials was observed only when the clinical outcome was measured with a newly developed outcome measure, the Systemic Lupus Erythematosus Responder Index. ${ }^{17}$

In summary, the results of the BLISS trials were modest but consistently favored a positive treatment effect of belimumab over placebo. The trials established that rigorous trials leading to positive outcomes can be performed in systemic lupus erythematosus, and clinical trial methodologies employed in studies of belimumab have important implications for future lupus trials. The fact that these trials excluded patients with active central nervous system (CNS) involvement and severe lupus nephritis limits the generalizability of results to these patient subsets.

Atacicept-Recently, a phase II trial of atacicept in combination with mycophenolate mofetil in lupus nephritis was suspended due to a high rate of severe infections; a phase II/ III trial of atacicept for patients with nonrenal lupus is ongoing. ${ }^{18}$ 


\section{Uncontrolled Studies}

Substantial clinical experience with off-label rituximab use has been accumulated in recent years, with nearly 200 cases included in open-label studies and small case series through 2008. ${ }^{19}$ Since 2009 , more than 700 additional patients have been reported. ${ }^{20-29}$ Thus, nearly 1000 patients with systemic lupus erythematosus have been enrolled in approximately 30 uncontrolled studies. Data from these investigations indicate rates of complete response to rituximab that range from $21 \%$ to $55 \%$, and overall responses from $60 \%$ to $91 \% .^{20-29}$

Significant clinical responses also have been observed in specific systemic lupus erythematosus subsets, including patients with lupus nephritis and CNS involvement. ${ }^{19} \mathrm{In}$ contrast to the case with rituximab, other therapies that target B cells specifically have not been employed outside of clinical trials to any significant degree.

\section{USING B-CELL-DEPLETING AGENTS IN CLINICAL PRACTICE}

\section{Clinical Indications}

The use of B-cell-depleting agents in clinical practice, overwhelmingly restricted to rituximab, is centered on 3 main clinical situations: patients who do not respond to standard therapy, patients who are intolerant to SOC therapy, and those with life-threatening presentations. The most common clinical scenario in which B-cell-targeted strategies are employed is in lupus nephritis (proliferative or membranous) that has proven refractory to cyclophosphamide and mycophenolate/azathioprine. Other frequent situations include the use of rituximab as second/third-line therapy in patients with refractory cytopenias, lupusrelated vasculitis, and CNS involvement, and even as first-line therapy in life-threatening situations (renal failure, pulmonary hemorrhage, myelitis).

There is no consensus on the dosage of rituximab in systemic lupus erythematosus. It is overwhelmingly used as an induction therapy; either as 4 weekly doses of $375 \mathrm{mg} / \mathrm{m}^{2}$ or 2 fortnightly doses of $1000 \mathrm{mg}$ (no study has compared the 2 dosages). With respect to belimumab, the recommended dosage will probably be $10 \mathrm{mg} / \mathrm{kg}$ administered intravenously over at least 120 minutes twice in the first month followed by monthly administration (therapy duration has not yet been defined).

\section{Re-treatment and Maintenance Therapy}

Information on the use of rituximab in the re-treatment of relapses is limited. The majority of uncontrolled studies show that flares often occur between 6 and 18 months after induction therapy, when circulating B cells have returned. ${ }^{30}$ Flares have been treated in a heterogeneous manner, with an increase in the dose of glucocorticosteroids, the addition of immunosuppressive agents, or re-treatment with rituximab. A recent study found that the efficacy of rituximab retreatment was $82 \%$ at 6 months and $65 \%$ at 12 months, and that the second cycle produces a more sustained clinical response than induction therapy. ${ }^{23}$ These authors also found that some patients who do not respond to the first cycle of rituximab responded to a second cycle. There is no reported information on the use of rituximab as a maintenance regimen, although some authors have suggested that repeated treatment every 6 months might induce the depletion of protective B-cell subsets involved in the reestablishment of self tolerance. ${ }^{31}$

\section{Role of Concomitant Therapies}

The effect of baseline therapies has proven to be the main problem in assessing significant differences between the B-cell-depleting agents and the placebo arms in lupus trials. Highdose glucocorticosteroids (EXPLORER) or the concomitant use of mycophenolate (LUNAR) have probably reduced the power of these studies to demonstrate the clinical advantage of adding rituximab to the SOC. ${ }^{32}$ The high treatment-response rates in the 
comparison groups in these trials, which have approached 50\%, underscore the need for larger trials to detect a relatively small clinical benefit, as recently demonstrated by the BLISS trials.

No controlled clinical studies have compared the benefits of the use of B-cell-depleting agents alone or in combination with other therapies (eg, glucocorticosteroids, immunosuppressants, or other biological agents). The majority of uncontrolled studies used an increased dosage of baseline oral glucocorticosteroids or intravenous glucocorticosteroids at the time of rituximab infusion. The most frequent reported immunosuppressant used in combination with rituximab is cyclophosphamide ( 2 infusions of $500-750 \mathrm{mg}$ ), but no controlled data have demonstrated superiority in comparison with rituximab alone. Concomitant oral immunosuppressive agents are often stopped when rituximab is given, although in some studies they are used continuously throughout. ${ }^{30}$ Therefore, there are no standardized recommendations on the use of concomitant immunosuppressive agents in patients with systemic lupus erythematosus treated with B-cell-depleting agents.

\section{TAILORING B-CELL-DEPLETING AGENTS ACCORDING TO INDIVIDUAL CHARACTERISTICS}

Agents that target B cells are not appropriate for all patients with systemic lupus erythematosus. Optimal uses of these treatment strategies would tailor therapies according to patients' individual characteristics, including ethnicity, organ involvement, or the immunological profile.

\section{Ethnicity}

Recently ethnicity has emerged as an important factor to be taken into account when response to immunosuppressive/biological agents is evaluated in patients with systemic lupus erythematosus. ${ }^{33,34}$ The EXPLORER trial showed that the highest percentage of clinical response to rituximab and the lowest placebo response was found in patients of Hispanic and African ancestry, ${ }^{6}$ which seems to be related to the more refractory disease often observed in these patients. A trend to benefit in Blacks with lupus nephritis treated with rituximab also has been recently reported in the LUNAR study. ${ }^{7,8}$ In the BLISS-52 trial, a trend to a better response to belimumab was observed in patients from eastern Europe in comparison with those from Asia and Latin America. ${ }^{14}$

\section{Organ Involvement}

Few studies have analyzed differences in the response to B-cell-depleting agents according to organ involvement. Little information is available on the specific response to rituximab of the different types of lupus nephritis, and some uncontrolled data suggest a higher rate of complete response in patients with refractory type III lupus nephritis compared with type IV. ${ }^{35}$ CNS involvement seems to have a good response to B-cell depletion in small uncontrolled studies. With respect to belimumab, data from the combined BLISS trials show significant improvement in the vascular and cutaneous Safety of Estrogen in Lupus Erythematosus National Assessment-Systemic Lupus Erythematosus Disease Activity Index (SELENA-SLEDAI) domains and in the musculoskeletal and mucocutaneous British Isles Lupus Assessment Group disease activity index (BILAG) domains; conversely, poorer results were observed in the hematological and renal domains. Contrasting results were found in the neurological domains (excellent results in the SLEDAI domain, poor results in the BILAG domain). ${ }^{36}$

There is no solid evidence that some manifestations are more amenable to treatment with rituximab than belimumab or vice versa. Patients included in the BLISS trials showed 
predominantly mucocutaneous and musculoskeletal features $(60 \%-80 \%),{ }^{16}$ while the largest international series analyzing the off-label use of rituximab in 317 patients (the IR-BISSLICC Registry) found that the major organ involvements leading to rituximab treatment were renal (50\%) and hematological involvement (21\%), with musculoskeletal and cutaneous features representing $<10 \%$ of cases. ${ }^{37}$ Additional studies of belimumab across a broader range of manifestations are required before this therapy can be employed with confidence in lupus nephritis, CNS disease, and other lupus features not adequately represented in the trials performed to date.

\section{Immunological Profile}

Some studies have suggested a differing response according to the baseline immunological profile. The effect of B-cell-depleting agents in reducing autoantibody production and normalizing complement levels has consistently been shown in the majority of trials and uncontrolled studies, and correlates with the greater efficacy of B-cell-depleting agents in patients with active disease. ${ }^{38}$ The combined results of the 2 BLISS trials showed a significantly better response to belimumab in patients with positive immunological markers ( $46 \%$ vs $29 \%$ in the placebo arm, $P=.006$ ). ${ }^{16}$ In contrast, some studies have suggested a poor response to rituximab in some specific immunological subsets, such as those with positive anti-Ro/SS-A or anti-Sm. ${ }^{39}$

\section{B-cell-depletion Predictors}

The degree and duration of B-cell depletion in systemic lupus erythematosus, although more variable and less predictable than in rheumatoid arthritis, often correlates with clinical response. Therefore, identification of baseline predictors of depletion may be useful (Table 3). ${ }^{39-44}$

\section{B-CELL-TARGETING THERAPIES: MAIN SIDE EFFECTS}

Infusion reactions are often mild to moderate, with severe reactions principally related to the lack of premedication. A recent study found a lower incidence of infusion-related reactions in patients with systemic lupus erythematosus in comparison with those with rheumatoid arthritis treated with rituximab; this may be due to the higher dosage of glucocorticosteroids used in systemic lupus erythematosus. ${ }^{45}$ The role of human antichimeric antibodies in infusion reactions is unclear, but might be associated with delayed serum sickness reactions.

The risk of severe infection is the main factor that should be considered when weighing the risks and benefits of using B-cell-depleting agents to treat systemic lupus erythematosus (Figure). Controlled trials using rituximab reported no significant increase in serious adverse effects, except for a higher rate of neutropenia $(8 \%)$ and herpes infection $(15 \%)$ in the EXPLORER trial. ${ }^{14}$ In contrast, uncontrolled data suggest that infection is the most frequent adverse event in the largest series and may be severe in some patients. ${ }^{19}$ Recent studies have estimated a rate of 63-66 severe infections per 1000 person-years. ${ }^{26,46}$ Infection of the lower respiratory or urinary tracts, together with bacteremia/sepsis, accounted for $75 \%$ of severe infections, most of which were caused by common bacteria.

The major concern about the safety of rituximab in systemic lupus erythematosus was raised in 2008 after reports of 2 patients who developed progressive multifocal leukoencephalopathy), although no additional cases have been reported from subsequent trials and large uncontrolled series. ${ }^{6-8,19-29,37}$ Progressive multifocal leukoencephalopathy is not exclusively restricted to rituximab use, ${ }^{47}$ and current evidence does not seem to provide a high-enough level of concern to warrant eliminating the off-label use of rituximab in systemic lupus erythematosus. 
Available controlled safety data for belimumab show that it is generally well tolerated and has a favorable safety profile. Commonly reported adverse events include headache, arthralgia, upper respiratory tract infections, urinary tract infections, and influenza. The BLISS-76 data showed $15 \%$ of infusion-related reactions, $7 \%$ of severe infections, and a discontinuation rate due to adverse events of $8 \% .{ }^{48}$

\section{FUTURE PERSPECTIVES}

The recent approval of belimumab for use in systemic lupus erythematosus suggests that biological agents will be increasingly used in the near future and will have a significant impact on the management of lupus patients. ${ }^{17,49,50}$ However, the efficacy and long-term safety of belimumab in clinical practice remains to be demonstrated ${ }^{2}$ (Table 4 ).

Patients with systemic lupus erythematosus display substantial heterogeneity in terms of Bcell homeostasis, whose functional consequences and implications for B-cell therapy remain to be determined. New treatments and the successful application of current ones will rest heavily on thorough understanding of these factors. This, in turn, may allow the customized application of biological agents targeting specific pathways or B-cell subsets in appropriate patient populations. Careful evaluations of the risk/benefit profiles of biologic agents in patients with systemic lupus erythematosus are essential, both in the context of RCTs and in off-label studies of the uses of these therapies. Finally, only real-life data will establish the balance between the clinical benefits and cost of B-cell-targeted therapies (estimated as at least \$20,000 annually); in countries where health provision is mainly private, patient access to these therapies may not be guaranteed.

\section{Acknowledgments}

Funding: None.

\section{References}

1. D'Cruz DP, Khamashta MA, Hughes GR. Systemic lupus erythematosus. Lancet. 2007; 369(9561): 587-596. [PubMed: 17307106]

2. Mitka M. Treatment for lupus, first in 50 years, offers modest benefits, hope to patients. JAMA. 2011; 305:1754-1755. [PubMed: 21540415]

3. Sanz I, Lee FE. B cells as therapeutic targets in SLE. Nat Rev Rheumatol. 2010; 6:326-337. [PubMed: 20520647]

4. Jacobi AM, Huang W, Wang T, et al. Effect of long-term belimumab treatment on B cells in systemic lupus erythematosus: extension of a phase II, double-blind, placebo-controlled, doseranging study. Arthritis Rheum. 2010; 62:201-210. [PubMed: 20039404]

5. Wallace DJ, Stohl W, Furie RA, et al. A phase II, randomized, double-blind, placebo-controlled, dose-ranging study of belimumab in patients with active systemic lupus erythematosus. Arthritis Rheum. 2009; 61:1168-1178. [PubMed: 19714604]

6. Merrill JT, Neuwelt CM, Wallace DJ, et al. Efficacy and safety of rituximab in moderately-toseverely active systemic lupus erythematosus: the randomized, double-blind, phase II/III systemic lupus erythematosus evaluation of rituximab trial. Arthritis Rheum. 2010; 62:222-233. [PubMed: 20039413]

7. Furie R, Looney J, Rovin B, et al. Efficacy and safety of rituximab in patients with proliferative lupus nephritis: results from the randomized, double-blind phase iii lunar study. Ann Rheum Dis. 2010; 69(Suppl 3):549.

8. Furie R, Rovin BH, Appel G, et al. Effect of rituximab on anti-double-stranded DNA antibody and C3 levels and relationship to response: results from the LUNAR trial. Ann Rheum Dis. 2010; 69(Suppl 3):550. [PubMed: 19778912] 
9. Mysler EF, Spindler AJ, Guzman R, et al. Efficacy and safety of ocrelizumab, a humanized antiCD20 antibody, in patients with active proliferative lupus nephritis (LN): results from the randomized, double-blind phase III BELONG study. Arthritis Rheum. 2010; 62 (Suppl):S606S607.

10. Wallace DJ, Hobbs K, Houssiau F, et al. Randomized controlled trials of epratuzumab (antiCD-22MAB targeting B cells) reveal clinically meaningful reductions in corticosteroid use with favorable safety profile in moderate and severe flaring SLE patients. Ann Rheum Dis. 2008; 67(Suppl II):212. [PubMed: 17526555]

11. Petri MA, Hobbs K, Gordon C, et al. Randomized controlled trials of epratuzumab (antiCD-22MAB targeting B cells) reveal clinically meaningful improvements in patients with moderate and severe SLE flares. Ann Rheum Dis. 2008; 67(Suppl II):53.

12. Wallace DJ, Kalunian KC, Petri MA, et al. Epratuzumab demonstrates clinically meaningful improvements in patients with moderate to severe systemic lupus erythematosus (SLE): results from EMBLEM, a phase IIb study. Arthritis Rheum. 2010; 62(Suppl):S605.

13. Petri M, Furie R, Merrill J, et al. Six-year experience with belimumab in patients with SLE. Ann Rheum Dis. 2011:Abstract THU0409.

14. Navarra SV, Guzmán RM, Gallacher AE, et al. BLISS-52 Study Group. Efficacy and safety of belimumab in patients with active systemic lupus erythematosus: a randomised, placebocontrolled, phase 3 trial. Lancet. 2011; 377:721-731. [PubMed: 21296403]

15. Vollenhoven RF, Schwarting A, Navarra S, et al. BLISS-52 and BLISS-76 Study Groups. Durability of response in SLE patients treated with belimumab in the phase 3 BLISS-52 and BLISS-76 studies. Ann Rheum Dis. 2011:Abstract THU0431.

16. D’Cruz D, Manzi S, Sánchez-Guerrero J, et al. BLISS-52 and BLISS-76 Study Groups. Belimumab reduced disease activity across multiple organ domains in patients with SLE: combined results from BLISS-52 and BLISS-76. Ann Rheum Dis. 2011:Abstract THU0421.

17. Sanz I, Yasothan U, Kirkpatrick P. Belimumab. Nat Rev Drug Discov. 2011; 10:335-336. [PubMed: 21532557]

18. Gunnarsson I, van Vollenhoven RF. Biologicals for the treatment of systemic lupus erythematosus? Ann Med. 2011 Apr 15. Epub ahead of print.

19. Ramos-Casals M, Soto MJ, Cuadrado MJ, Khamashta MA. Rituximab in systemic lupus erythematosus: a systematic review of off-label use in 188 cases. Lupus. 2009; 18:767-776. [PubMed: 19578100]

20. Vital EM, Dass S, Buch MH, et al. Rituximab responses in systemic lupus erythematosus explained by B cell biomarkers. Arthritis Rheum. 2011 May 25. Epub ahead of print. 10.1002/art. 30466

21. Tony HP, Burmester G, Schulze-Koops H, et al. Safety and clinical outcomes of rituximab therapy in patients with different autoimmune diseases: experience from a national registry (GRAID). Arthritis Res Ther. 2011; 13:R75. [PubMed: 21569519]

22. Tesfa1 D, Ajeganova S, Hägglund1 $\mathrm{H}$, et al. Late-onset neutropenia following rituximab therapy in rheumatic diseases: association with B-lymphocyte depletion and infections. Arthritis Rheum. 2011 May 10. Epub ahead of print. 10.1002/art.30427

23. Turner-Stokes T, Lu TY, Ehrenstein MR, Giles I, Rahman A, Isenberg DA. The efficacy of repeated treatment with B-cell depletion therapy in systemic lupus erythematosus: an evaluation. Rheumatology (Oxford). 2011 Mar 12. Epub ahead of print.

24. Galarza-Maldonado C, Kourilovitch MR, Molineros JE, et al. The administration of low doses of rituximab followed by hydroxychloroquine, prednisone and low doses of mycophenolate mofetil is an effective therapy in Latin American patients with active systemic lupus erythematosus. Autoimmun Rev. 2010; 10:108-111. [PubMed: 20804861]

25. Ramos-Casals M, García-Hernández FJ, de Ramón E, et al. BIOGEAS Study Group. Off-label use of rituximab in 196 patients with severe, refractory systemic autoimmune diseases. Clin Exp Rheumatol. 2010; 28:468-476. [PubMed: 20525449]

26. Terrier B, Amoura Z, Ravaud P, et al. Club Rhumatismes et Inflammation. Safety and efficacy of rituximab in systemic lupus erythematosus: results from 136 patients from the French 
AutoImmunity and Rituximab registry. Arthritis Rheum. 2010; 62:2458-2466. [PubMed: 20506527]

27. Catapano F, Chaudhry AN, Jones RB, Smith KG, Jayne DW. Long-term efficacy and safety of rituximab in refractory and relapsing systemic lupus erythematosus. Nephrol Dial Transplant. 2010; 25:3586-3592. [PubMed: 20466686]

28. Garcia-Carrasco M, Mendoza-Pinto C, Sandoval-Cruz M, et al. Anti-CD20 therapy in patients with refractory systemic lupus erythematosus: a longitudinal analysis of 52 Hispanic patients. Lupus. 2010; 19:213-219. [PubMed: 19965944]

29. Melander C, Sallée M, Trolliet P, et al. Rituximab in severe lupus nephritis: early B-cell depletion affects long-term renal outcome. Clin J Am Soc Nephrol. 2009; 4:579-587. [PubMed: 19261822]

30. Favas C, Isenberg DA. B-cell-depletion therapy in SLE-what are the current prospects for its acceptance? Nat Rev Rheumatol. 2009; 5:711-716. [PubMed: 19946298]

31. Sanz I. The conundrum of B cell depletion in SLE. Nat Rev Rheumatol. 2009; 5:304-305. [PubMed: 19491910]

32. Bruce IN. Re-evaluation of biologic therapies in systemic lupus erythematosus. Curr Opin Rheumatol. 2010; 22:273-277. [PubMed: 20177385]

33. Rivera TL, Belmont HM, Malani S, et al. Current therapies for lupus nephritis in an ethnically heterogeneous cohort. J Rheumatol. 2009; 36:298-305. [PubMed: 19040310]

34. Isenberg D, Appel GB, Contreras G, et al. Influence of race/ethnicity on response to lupus nephritis treatment: the ALMS study. Rheumatology (Oxford). 2010; 49:128-140. [PubMed: 19933596]

35. Ramos-Casals M, Diaz-Lagares C, Soto-Cardenas MJ, et al. Rituximab therapy in lupus nephritis: current clinical evidence. Clin Rev Allergy Immunol. 2011; 40:159-169. [PubMed: 20419398]

36. Merrill JT, Wallace DJ, Furie RA, et al. Five-year experience with belimumab, a BLyS-specific inhibitor, in patients with systemic lupus erythematosus (SLE). Arthritis Rheum. 2010; 62(Suppl):S608.

37. van Vollenhoven RF, Jacobsen S, Wallace D, et al. SLICC/IRBIS group. Biologics use in SLE in 18 centers - data from the international registry for biologics in SLE (IRBIS). Ann Rheum Dis. 2011:Abstract THU0432.

38. Lateef A, Petri M. Biologics in the treatment of systemic lupus erythematosus. Curr Opin Rheumatol. 2010; 22:504-509. [PubMed: 20502332]

39. Cambridge G, Isenberg DA, Edwards JC, et al. B cell depletion therapy in systemic lupus erythematosus: relationships among serum B lymphocyte stimulator levels, autoantibody profile and clinical response. Ann Rheum Dis. 2008; 67:1011-1016. [PubMed: 17962238]

40. Croca SC, Isenberg DA. Analysis of the use of rituximab in the treatment of lupus nephritis. Arthritis Rheum. 2010; 62(Suppl):S479.

41. Croca SC, Isenberg DA. Immunoglobulin levels as potential predictors of response to B-cell depletion. Arthritis Rheum. 2010; 62(Suppl):S195.

42. Anolik JH, Campbell D, Felgar RE, et al. The relationship of FcgammaRIIIa genotype to degree of B cell depletion by rituximab in the treatment of systemic lupus erythematosus. Arthritis Rheum. 2003; 48:455-459. [PubMed: 12571855]

43. Jónsdóttir T, Gunnarsson I, Risselada A, Henriksson EW, Klareskog L, van Vollenhoven RF. Treatment of refractory SLE with rituximab plus cyclophosphamide: clinical effects, serological changes, and predictors of response. Ann Rheum Dis. 2008; 67:330-334. [PubMed: 17827182]

44. Sutter JA, Kwan-Morley J, Dunham J, et al. A longitudinal analysis of SLE patients treated with rituximab (anti-CD20): factors associated with B lymphocyte recovery. Clin Immunol. 2008; 126:282-290. [PubMed: 18226586]

45. Conti F, Ceccarelli F, Perricone C, et al. Rituximab infusion-related adverse event rates are lower in patients with systemic lupus erythematosus than in those with rheumatoid arthritis. Rheumatology (Oxford). 2011; 50:1148-1152. [PubMed: 21278072]

46. Díaz-Lagares C, Pérez-Alvarez R, García-Hernández FJ, et al. BIOGEAS Study Group. Rates of and risk factors for severe infections in patients with systemic autoimmune diseases receiving biological agents off-label. Arthritis Res Ther. 2011; 13:R112. [PubMed: 21745378] 
47. Molloy ES, Calabrese LH. Progressive multifocal leukoencephalopathy associated with biologic and synthetic DMARD therapy in rheumatic diseases: an analysis of the FDA adverse event reporting system database. Arthritis Rheum. 2010; 62(Suppl):S292.

48. Furie R, Zamani O, Wallace D, et al. Belimumab, a BLyS-specific inhibitor, reduced disease activity and severe flares in seropositive SLE patients: BLISS-76 study results through Wk 76. Arthritis Rheum. 2010; 62(Suppl):S606.

49. Cancro MP, D’Cruz DP, Khamashta MA. The role of B lymphocyte stimulator (BLyS) in systemic lupus erythematosus. J Clin Invest. 2009; 119:1066-1073. [PubMed: 19411764]

50. Engel P, Gómez-Puerta JA, Ramos-Casals M, Lozano F, Bosch X. Therapeutic targeting of B cells for rheumatic autoimmune diseases. Pharmacol Rev. 2011; 63:127-156. [PubMed: 21245206] 


\section{CLINICAL SIGNIFICANCE}

- Belimumab is the first drug licensed for use in systemic lupus erythematosus (SLE) in more than 50 years.

- The use of B-cell-depleting agents in clinical practice is centered on SLE patients with refractory/life-threatening disease.

- Careful evaluations of the risk/benefit profiles of biologic agents in SLE are essential.

- Biological agents will be increasingly used in the near future and will have a significant impact on the management of SLE patients. 


\begin{tabular}{|c|c|c|c|c|c|c|}
\hline Agent & $\begin{array}{l}\text { Name of } \\
\text { trial }\end{array}$ & $\begin{array}{l}\text { SLE activity criteria } \\
\text { for inclusion }\end{array}$ & $\begin{array}{l}\text { Response measurement } \\
\text { instrument }\end{array}$ & Follow-up & $\begin{array}{l}\text { \% of benefit over } \\
\text { placebo }\end{array}$ & $\begin{array}{l}\text { \% of severe } \\
\text { infection }\end{array}$ \\
\hline Belimumab & BLISS-52 & SELENA-SLEDAI $\geq 6$ & SRI & 52 weeks & $\begin{array}{l}7 \% * \\
14 \% *\end{array}$ & $\begin{array}{l}8 \% \\
4 \% \\
6 \%\end{array}$ \\
\hline Belimumab & BLISS-76 & SELENA-SLEDAI $\geq 6$ & SRI & 52 weeks & $\begin{array}{l}7 \% \\
9 \% *\end{array}$ & \begin{tabular}{l|l}
$7 \%$ \\
$7 \%$ \\
$6 \%$
\end{tabular} \\
\hline Belimumab & BLISS-76 & SELENA-SLEDAI $\geq 6$ & SRI & 76 weeks & $\begin{array}{l}6 \% \\
7 \%\end{array}$ & \begin{tabular}{|l|l}
$6 \%$ & \\
$7 \%$ \\
$5 \%$
\end{tabular} \\
\hline Rituximab & EXPLORER & $\begin{array}{l}\text { BILAG } A \geq 1 \text { or } \\
\text { BILAG } B \geq 2\end{array}$ & BILAG & 52 weeks & $1 \%^{\mathrm{a}}$ & $9.5 \%$ \\
\hline Rituximab & LUNAR & LN class III/IV & Renal response & 52 weeks & $11 \%^{\mathrm{a}}$ & $\begin{array}{l}4 \% \\
1 \%\end{array}$ \\
\hline Epratuzumab & EMBLEM & $\begin{array}{l}\text { BILAG } 2004 \mathrm{~A} \geq 1 \text { or } \\
\text { BILAG } 2004 \mathrm{~B} \geq 2\end{array}$ & CRI & 12 weeks & $25 \% *$ & Not detailed \\
\hline Ocrelizumab & BELONG & LN class III/IV & Renal response & 48 weeks & $\begin{array}{l}11 \% \\
13 \%\end{array}$ & $\begin{array}{r}25 \% \\
17 \% \\
14 \%\end{array}$ \\
\hline
\end{tabular}

Figure.

Summary of the main randomized controlled trials in systemic lupus erythematosus (SLE). $\%$ of benefit over placebo: low dose (light brown) and high dose (dark brown). \% of severe infection: low dose (light brown), high dose (dark brown), and placebo (white). *Statistical

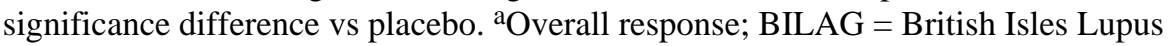
Assessment Group disease activity index; SELENA = Safety of Estrogen in Lupus Erythematosus National Assessment; SLEDAI = Systemic Lupus Erythematosus Disease Activity Index; SRI = Systemic Lupus Erythematosus Responder Index; CRI = Combined Responder Index; LN = lupus nephritis; SLE: systemic lupus erythematosus. 


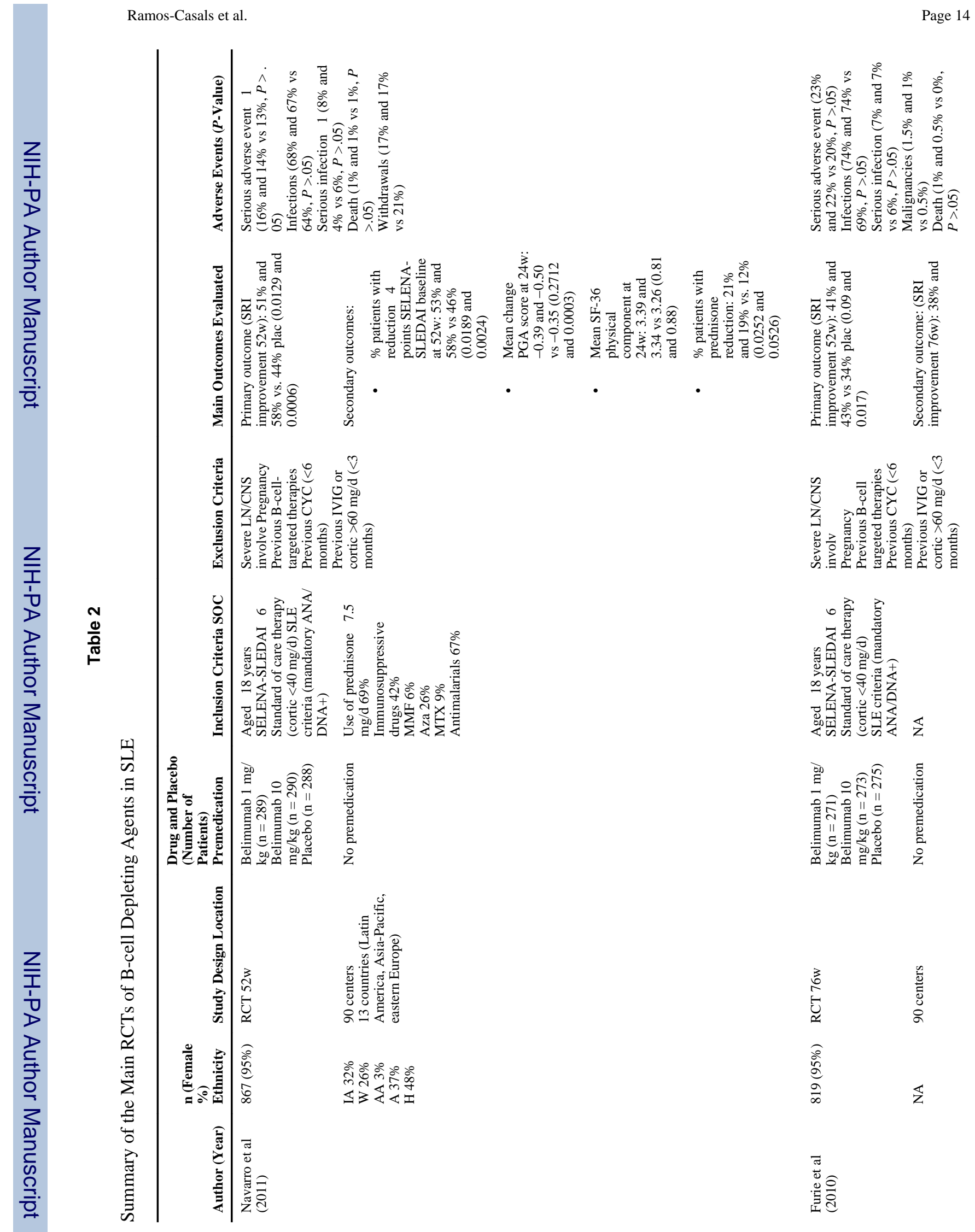

Am J Med. Author manuscript; available in PMC 2014 February 15 


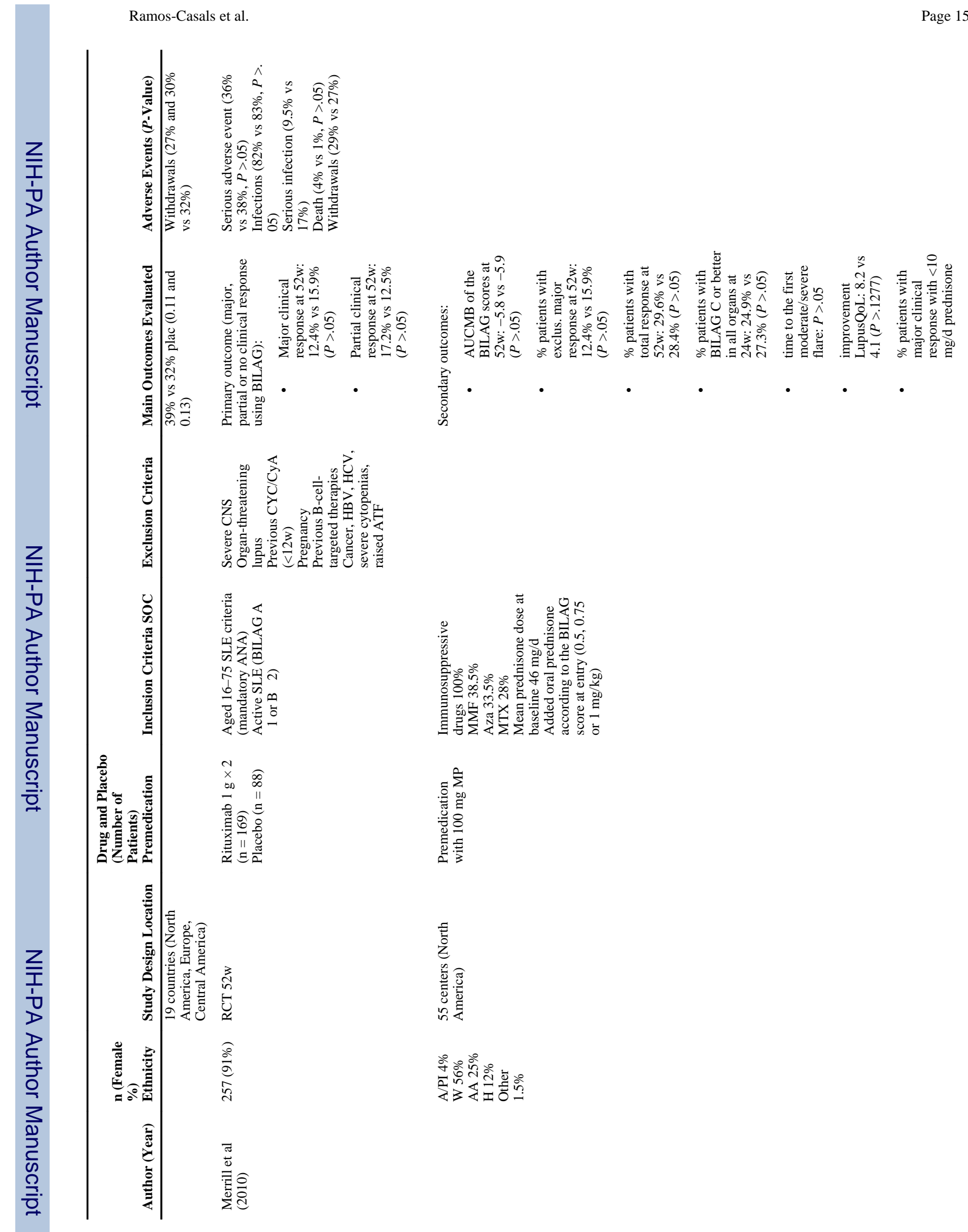




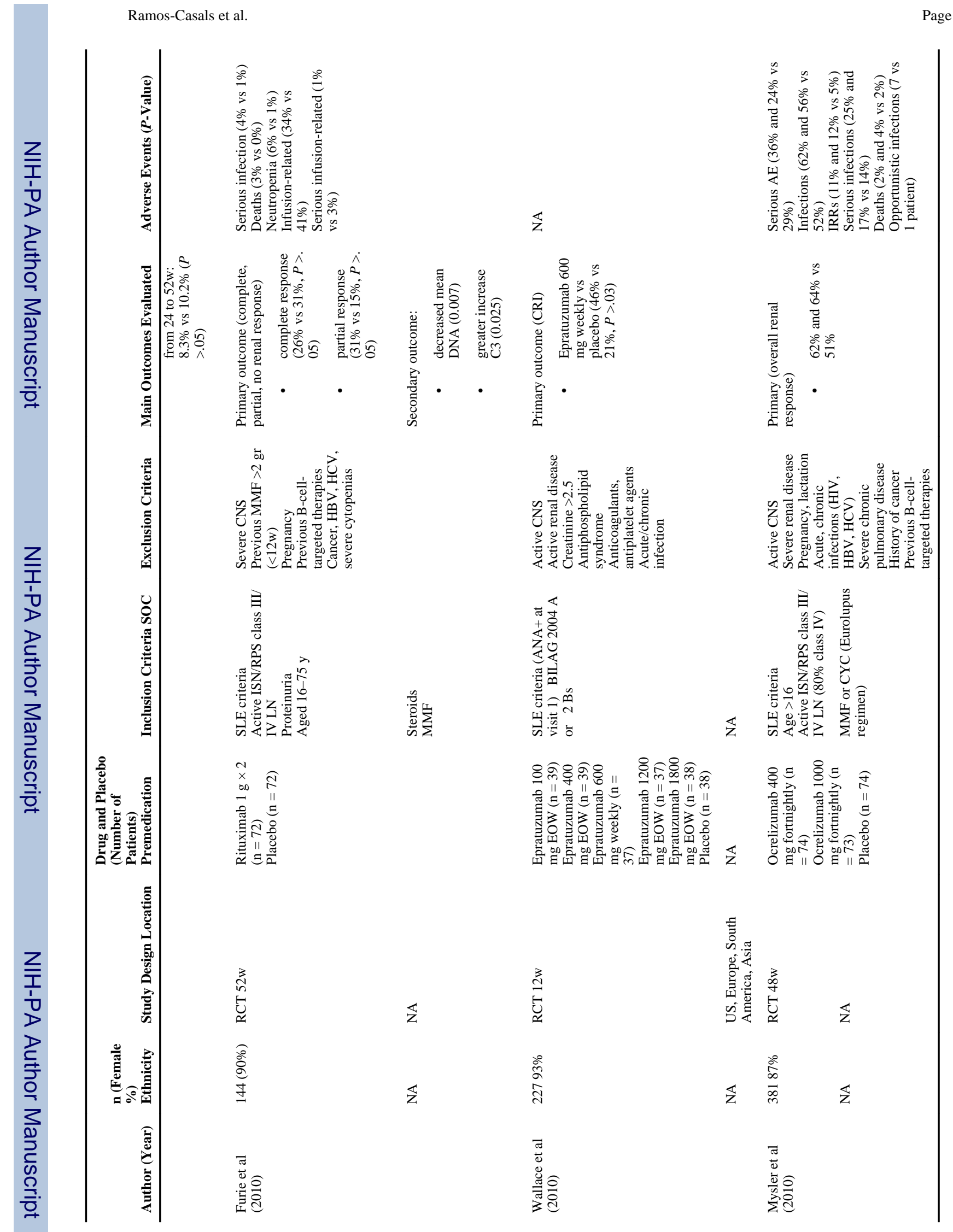




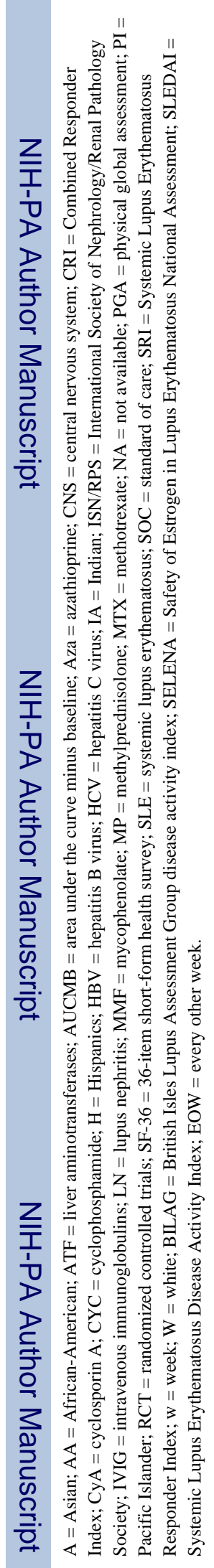




\section{Table 3}

Clinical, Immunological and Genetic Variables That May Influence B-cell Depletion in Patients with SLE Treated with Rituximab ${ }^{39-44}$

\begin{tabular}{ll}
\hline Variables & Effect on B-cell Depletion \\
\hline Afro-Caribbean origin & Early repopulation $(<6$ months $)$ \\
Anti-Sm+ carriers & Early repopulation ( $<6$ months $)$ \\
Low IgA levels at baseline & Depletion $>6$ months \\
Low IgG levels at baseline & Depletion $>6$ months \\
Serum rituximab levels & Correlation with $\%$ of peripheral B cells at 2 months \\
Fc $\gamma r I I I a$ genotype & Independent predictor of B-cell depletion \\
High numbers of CD19+ B lymphocytes at baseline & Shorter depletion time \\
\hline
\end{tabular}

$\operatorname{IgA}=$ immunoglobulin $\mathrm{A} ; \mathrm{IgG}=$ immunoglobulin $\mathrm{G} ; \mathrm{SLE}=$ systemic lupus erythematosus. 
